\title{
Early initiation and regular breast milk expression reduces risk of lactogenesis II delay in at-risk Singaporean mothers in a randomised trial
}

\author{
Doris $\underline{\text { Fok }}^{1}$, FILCA, Izzuddin Mohd $\underline{\text { Aris }}^{2}$, PhD, Jiahui $\underline{H o}^{1}$, BSSc, Yiong-Huak $\underline{\operatorname{Chan}}^{3}$, PhD, Mary Rauff' ${ }^{1}$, FRCoG, James KC $\underline{\operatorname{Lui}}^{4}$, PhD,
} Mark D $\underline{\text { Cregan }}^{5}$, PhD, Peter Hartmann ${ }^{5}$, PhD, Yap Seng $\underline{\text { Chong }}^{1,2}$, MD, Citra NZ Mattar ${ }^{1}$, MRANZCOG, PhD

\begin{abstract}
INTRODUCTION Lactogenesis II (Lall) failure can be prevented in at-risk mothers with simple proactive interventions. In a randomised trial, we investigated the efficacy of early and regular breast milk expression in establishing Lall, using an electric double-breast pump.

METHODS Mothers with uncomplicated singleton deliveries were randomised to intervention $(n=31)$ or control $(n=29)$ groups. The former commenced breast milk expression with an electric pump within one hour of delivery and maintained regular expression with direct breastfeeding. Control mothers directly breastfed without regular pump expression. Expressed milk volumes were analysed for citrate, lactose, sodium and protein.

RESULTS Median time of Lall was Day 3 (interquartile range [IQR] 1 day) with intervention and on Day 4 (IQR 1 day) among controls $(p=0.03)$. Biochemical steady-state concentrations were achieved around early Day 4 (sodium, total protein) and Days 4-5 (citrate, lactose). Sodium, protein and lactose levels were similar in both groups over seven days, at $5.80 \mathrm{mM}, 0.68 \mathrm{mM}$ and $-13.38 \mathrm{mM}$, respectively. Mean daily milk volume with intervention was $73.9 \mathrm{~mL}$ on Day 3 and $225.2 \mathrm{~mL}$ on Day 7 , greater than controls $(25.4 \mathrm{~mL}$ on Day 3 and $69.2 \mathrm{~mL}$ on Day $7 ; \mathrm{p}<0.2$ ). Mean infant weights were similar on Day 8 at 3,477 g with intervention and 3,479 g among controls.

CONCLUSION Lall is established by postnatal Day 3 with early initiation of regular breast milk expression, a useful intervention for mothers at risk of early-onset breastfeeding failure.
\end{abstract}

Keywords: breast pump, breastfeeding initiation, lactation delay, lactogenesis, regular expression

\section{INTRODUCTION}

Exclusive breastfeeding (EBF) rates in developed countries are currently below the proposed global target of at least $50 \%$ during the first six postnatal months, as determined by the United Nations System Standing Committee on Nutrition in 2012. About one-third of mothers practise EBF, with lower rates expected in developed countries. ${ }^{(1)}$ Although the incidence of initial breastfeeding reached 83\% between 2005 and 2010 in the United Kingdom (UK), only $31 \%$ of babies remained on EBF at six weeks, ${ }^{(2)}$ while in Singapore in 2013, only $1 \%$ of babies remained on EBF at six months despite a high initiation rate of $99 \% .{ }^{(3)}$ Given the many established health benefits, a concerted effort to overcome identifiable obstacles to sustained breastfeeding is urgently required, particularly in at-risk mothers. ${ }^{(4,5)}$

Lactogenesis II (Lall), also known as secretory activation, ${ }^{(6)}$ is the onset of copious milk production, which typically occurs at 40-72 postnatal hours, ${ }^{(7)}$ and increases rapidly and plateaus at $\sim 96$ postnatal hours ${ }^{(8)}$ Conditions associated with delayed Lall

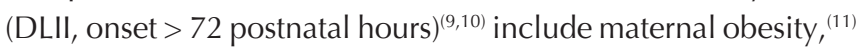
insulin-dependent diabetes mellitus, ${ }^{(12)}$ preterm delivery ${ }^{(13)}$ and Caesarean section. ${ }^{(14,15)}$ As DLII is an important contributing factor to early breastfeeding cessation, ${ }^{(16,17)}$ interventions that reduce DLII should be implemented in all maternity units.
Evidence suggests that breastfeeding initiation within a few hours of birth decreases Lall failure in at-risk women. ${ }^{(18,19)}$ Because the initial interaction between mother and baby may be iatrogenically delayed owing to operative delivery or perinatal complications, ${ }^{(20-24)}$ a sub-group of at-risk women can be identified for early assessment and intervention in the maternity unit. Milk expression with electric breast pumps (EBPs) is a common practice in Singapore, with $68.5 \%$ of Chinese mothers in a local study using pump-expressed breast milk at three months. ${ }^{(25)}$ EBPs are often utilised for milk collection and storage for future feeds or to determine the volume of milk fed to the infant. Early initiation independently contributes to improved breastfeeding rates at six months. ${ }^{(26)}$ In postpartum situations that physically prevent direct breast feeding, early breast milk expression by at-risk mothers may fulfil a similar role.

In this randomised study, we investigated the hypothesis that DLII is reduced when EBP expression is initiated early and performed regularly after delivery. Specifically, we determined the time at which Lall was successfully established by coupling simple observational assessment with biochemical validation, and analysed total daily milk volume expressed and infant weight change between the start and end of the first postnatal week.

\footnotetext{
${ }^{1}$ Department of Obstetrics and Gynaecology, Yong Loo Lin School of Medicine, National University of Singapore, ${ }^{2}$ Singapore Institute for Clinical Sciences (SICS), Agency for Science, Technology and Research (A*STAR), ${ }^{3}$ Biostatistics Unit, Yong Loo Lin School of Medicine, National University of Singapore, Singapore, ${ }^{4}$ Proteomics International, Broadway, Nedlands, ${ }^{5}$ School of Chemistry and Biochemistry, The University of Western Australia, Australia

Correspondence: Dr Citra NZ Mattar, Assistant Professor, Department of Obstetrics and Gynaecology, Yong Loo Lin School of Medicine, National University of Singapore, National University Health System, Singapore 119228. citramattar@nus.edu.sg
} 


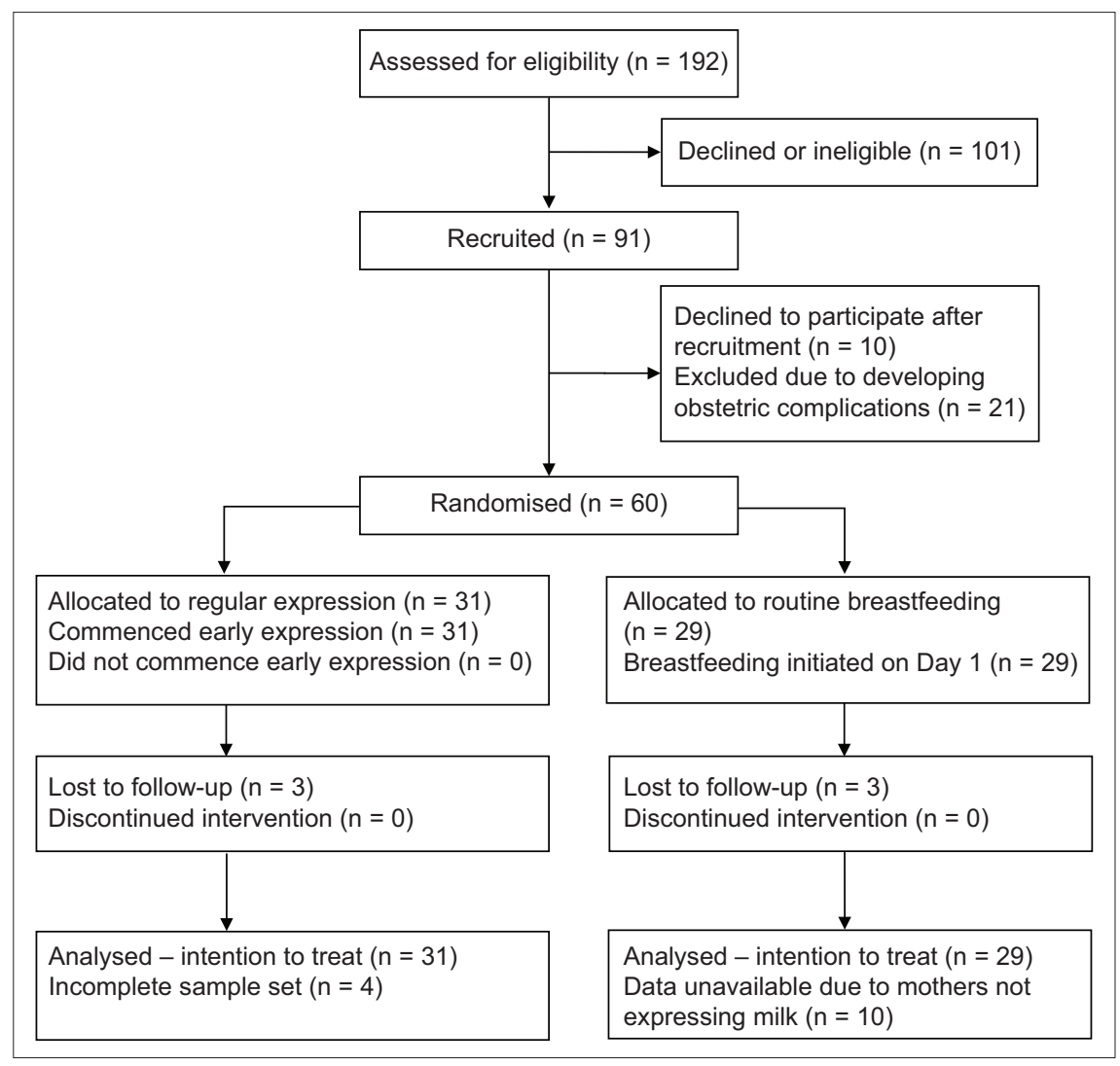

Fig. 1 Flowchart shows the enrolment and randomisation processes.

\section{METHODS}

This study was funded by a National Healthcare Group (NHG) research grant, NHG RPR 04027, and was conducted with the approval of the Domain Specific Review Board (DSRBD/04/119). The trial was registered with ClinicalTrials.gov (NCT00393640). Recruitment and interventions were initiated at the National University Hospital (NUH), Singapore.

Studies on lactation following preterm delivery have shown compromised initiation of lactation in up to $82 \%$ of mothers. ${ }^{(13)}$ Based on this, we required a sample size of 30 women in each arm to show a $50 \%$ reduction in DLII incidence with a significance level of 0.05 and a power of $90 \%$. We recruited women with term deliveries ( $\geq 37$ completed weeks of gestation) who met the eligibility criteria for the trial: minimum age of 21 years, a desire to breastfeed, agreement to use the breast pump and no contraindications to breastfeeding. All patients provided written informed, independent consent to trial participation and were subsequently randomised by minimisation of the stratification factors of previous live births and breastfeeding experience (Fig. 1).

Patients with uncomplicated singleton deliveries were assigned to the intervention or control groups at the time of recruitment according to a computer-generated randomised list, but were only informed of their specific assignments after delivery. Exclusion criteria included maternal diabetes mellitus, hyperprolactinaemia, congenital fetal anomalies and multiple pregnancies. A standard questionnaire was used to record demographic information, details of the home environment and attitudes towards breastfeeding. All mothers had access to literature on the benefits of breastfeeding and were given the $\mathrm{NUH}$ Breastfeeding Booklet. The intervention group had a strict regimen of breast milk expression starting within one hour of delivery and were instructed to continue two- to three-hourly expression throughout the week, in addition to direct breastfeeding whenever desired. Participants were visited by the research coordinator within the first postnatal hour and educated on the use of a double-flange EBP (Ameda Egnell or Pump In Style ${ }^{\circledR}$, Medela AG, Baar, Switzerland). Simultaneous breast expression was performed six to eight times during the day and at least once at night, with each session limited to 15 minutes. Participants were taught to feed expressed breast milk (EBM) to infants by spoon or cup to avoid nipple confusion. They were also encouraged to breastfeed directly after expression. Mothers in the control group were given appropriate instructions on expression and storage of breast milk and were allowed independence in determining their feeding schedules (for both direct and expressed milk feeds).

In addition to providing the required expressed milk samples, mothers from both control and intervention groups were encouraged to breastfeed or express milk according to hospital recommendations and were expected to use their own breast pumps. The primary outcome measure was maternal perception of copious milk flow, the most commonly used indicator of Lall. Secondary outcome measures included biochemical changes in breast milk, volume of breast milk expressed daily, and type and 
frequency of breastfeeding. Mothers in both groups collected breast milk samples twice daily from the day of delivery for the first week. The first 24 hours counted as Day 1, and total daily breast milk volume was recorded over each subsequent 24-hour period for seven days. All mothers recorded EBM volume using a calibrated syringe, after which the EBM was used immediately or stored appropriately for future use. Mothers recorded the total volume expressed at the end of each 24-hour period in their breastfeeding diaries. $1 \mathrm{~mL}$ of milk was extracted from the first and last yields of the day for biochemical analyses. Syringes and polypropylene containers were used to aliquot and store EBM samples at $-20^{\circ} \mathrm{C}$ in the mother's home freezer until collection at the end of the week. Long-term samples were stored at $-80^{\circ} \mathrm{C}$.

Neonatal feeding data consisting of childbirth, perinatal and infant feeding details were collected via standardised forms at the first postnatal visit conducted at Week 1. In the first week, participants prospectively recorded in their breastfeeding diaries their perception of when their milk first came in (as an indicator of Lall), (27) EBM volume, frequency and duration of direct breastfeeding, other fluids fed and breastfeeding problems encountered (if any). Exclusive breast milk feeding was defined as feeding only breast milk (expressed or direct), while mixed feeding was defined as giving breast milk with formula in any amounts. Infant weights were recorded using a Seca 334 scale (seca Deutschland, Hamburg, Germany) on Days 2 and 8.

For biochemical analysis of samples, batch testing of EBM samples was performed to determine the concentrations of total protein, citrate, lactate and sodium. Milk samples were thawed at room temperature, centrifuged and defatted as described by Arthur et al. ${ }^{(7)}$ Total protein, sodium, citrate and lactose concentrations were assayed using methods previously described. ${ }^{(13)}$

Descriptive statistics were reported as mean \pm standard deviation for continuous variables and percentages for categorical variables. Differences between groups were assessed using chisquare analyses, apart from infant weight, which was assessed by independent sample $t$-test. We serially examined milk volume and the corresponding biochemical profile (i.e. sodium, lactose, citrate and protein concentrations) over seven days with linear mixedeffects models that took into account the correlation between repeated measures from the same individual and incomplete outcome data, using the assumption that it was missing at random. Maximum likelihood estimation was used and a diagonal working covariance matrix for random effects variables (intercept and slope) was chosen. The Akaike information criterion statistic facilitated the model selection, and final models included timeintervention interactions to estimate the change in milk volume and biochemical profile over time between intervention groups. All statistical analysis was conducted using IBM SPSS Statistics version 19.0 (IBM Corp, Armonk, NY, USA) and GraphPad Prism Version 6.04 (GraphPad Software, La Jolla, CA, USA).

\section{RESULTS}

A total of 192 women were approached for this study and 91 were initially recruited. After excluding women with pregnancies complicated by diabetes mellitus, hyperprolactinaemia and congenital anomalies, or with multifetal pregnancies, 60 mothers were randomised into the intervention group $(n=31)$ and control group ( $\mathrm{n}=29$, Fig. 1 ). Most of the participants in both groups were Chinese $(n=27)$ or Malay $(n=27)$. Table I summarises the characteristics of the participants. There were no significant differences between the two groups in terms of demographics (age, parity, body mass index, education, occupation, household income and pre-pregnancy medical conditions), perinatal factors (mode of delivery, skin-to-skin time and neonatal interventions) or breastfeeding practices and attitudes (intention to breastfeed, attendance at antenatal classes, advice from family and education received from primary caregivers).

Mothers in the intervention group described copious milk flow from Day 2 onwards, except for two mothers who recorded this event in the first half (Day 1.0) and second half (Day 1.5) of Day 1 , with the median time being Day 3 (72 completed hours) (interquartile range 1 day). Among the control group, copious milk flow was first reported on Day 2, with the median time being Day 4, when 14 mothers reported copious milk flow (Fig. 2a). The maternal perception of Lall in the intervention group was statistically significant $(p=0.03)$. Intervention group mothers were $64 \%$ more likely to have milk come in by Day 3 compared with controls, producing a likelihood ratio for non-delayed Lall of 1.64 (95\% confidence interval $[\mathrm{CI}] 1.0-2.7, \mathrm{p}<0.05)$. By the end of the first 72 hours (i.e. Day 3), 18 mothers in the intervention group had reported milk flow (vs. nine in the control group, $p=0.19$ ). After Day 3, 13 intervention group mothers had reported milk flow, compared to 20 control group mothers $(p=0.80)$. The majority of mothers in the control group experienced copious milk flow by the evening of Day 4 .

When mothers of different races were compared, Chinese and Malay mothers demonstrated comparable time points at which copious milk flow was reported (Fig. 2b). A higher proportion of Malay mothers reported copious milk flow by Day $3(80.8 \%$, $21 / 26)$ compared to Chinese mothers $(66.7 \%, 16 / 24)$. The median time of milk coming in among Malay mothers was the first half of Day 3, whereas among Chinese mothers, it was the second half of Day 3. These differences were not significant $(p=0.19)$.

Regarding the biochemical levels of the two groups, sodium and total protein concentrations peaked on Day 1 and reached steady state at around early Day 4, while citrate and lactose levels achieved steady state at Days 4-5. Over the seven-day period, when mean concentrations of the biochemical markers were compared between the intervention and control groups, we found no significant differences in sodium, protein and lactose levels, which were $5.80(95 \% \mathrm{Cl}-3.03,14.62) \mathrm{mM}, 0.68(95 \% \mathrm{Cl}-2.38$, $3.75) \mathrm{mM}$ and $13.38(95 \% \mathrm{Cl}-28.73,1.97) \mathrm{mM}$, respectively (Figs. 3a, b \& d). Mean citrate concentrations were, however, significantly higher in the intervention group at $0.84(95 \% \mathrm{Cl}$ $0.16,1.51) \mathrm{mM}$ (Fig. 3c). Differences between the groups at each time point were negligible.

Mothers in both groups fed their infants either breast milk, alone or supplemented with water only, or a combination of breast milk and milk formula (Fig. 4). When infant feeding practices and infant weight were examined, more mothers in both 
Table I. Demographic data of women enrolled into the regular expression and routine breastfeeding groups.

\begin{tabular}{|c|c|c|c|}
\hline \multirow[t]{2}{*}{ Variable } & \multicolumn{2}{|c|}{ No. (\%) } & \multirow[t]{2}{*}{ p-value } \\
\hline & Regular expression $(n=31)$ & Routine breastfeeding $(n=29)$ & \\
\hline Ethnicity & & & 0.275 \\
\hline Malay & $11(35.5)$ & $16(55.2)$ & \\
\hline Chinese & $17(54.8)$ & $10(34.5)$ & \\
\hline Indian & $2(6.5)$ & $3(10.3)$ & \\
\hline Other & $1(3.2)$ & $0(0)$ & \\
\hline Age (yr) & & & 0.509 \\
\hline$\geq 35$ & $7(22.6)$ & $4(13.8)$ & \\
\hline$<35$ & $24(77.4)$ & $25(86.2)$ & \\
\hline Body mass index & & & 0.409 \\
\hline$<25$ & $29(93.5)$ & $25(86.2)$ & \\
\hline$\geq 25$ & $2(6.5)$ & $4(13.8)$ & \\
\hline Occupation & & & 0.501 \\
\hline Professional & $17(54.8)$ & $12(41.4)$ & \\
\hline Technical & $6(19.4)$ & $5(17.2)$ & \\
\hline Self-employed & $0(0)$ & $1(3.4)$ & \\
\hline Homemaker & $8(25.8)$ & $11(37.9)$ & \\
\hline Household income (SGD) & & & 0.734 \\
\hline$\leq 60,000$ & $19(61.3)$ & $19(65.5)$ & \\
\hline$>60,000$ & $12(38.7)$ & $10(34.5)$ & \\
\hline Type of housing & & & 0.605 \\
\hline Public housing & $24(77.4)$ & $24(82.8)$ & \\
\hline Private housing & $7(22.6)$ & $5(17.2)$ & \\
\hline \multicolumn{4}{|l|}{ Education } \\
\hline Secondary school or below & $11(35.5)$ & $12(41.4)$ & 0.559 \\
\hline High school/college & $9(29.0)$ & $5(17.2)$ & \\
\hline University & $11(35.5)$ & $12(41.4)$ & \\
\hline Household size & & & 0.951 \\
\hline Nuclear & $19(61.3)$ & $18(62.1)$ & \\
\hline Extended & $12(38.7)$ & $11(37.9)$ & \\
\hline Antenatal factors & & & 0.118 \\
\hline Primigravida & $7(22.6)$ & $12(41.4)$ & \\
\hline Multigravida & $24(77.4)$ & $17(58.6)$ & \\
\hline Medical conditions & & & 0.648 \\
\hline Yes & $5(16.1)$ & $6(20.7)$ & \\
\hline No & $26(83.9)$ & $25(86.2)$ & \\
\hline Breastfeeding experience & & & 0.315 \\
\hline Yes & $20(64.5)$ & $15(51.7)$ & \\
\hline No & $11(35.5)$ & $14(48.3)$ & \\
\hline \multicolumn{4}{|l|}{ Breastfeeding plan prior to birth } \\
\hline Intent to breastfeed & $31(100.0)$ & $29(100.0)$ & 0.475 \\
\hline Antenatal classes & $7(22.6)$ & $3(10.3)$ & 0.302 \\
\hline Pro-breastfeeding advice from family/friends & $29(93.5)$ & $23(79.3)$ & 0.140 \\
\hline Intent to return to work & $22(71.0)$ & $18(62.1)$ & 0.313 \\
\hline Received breastfeeding advice & $29(93.5)$ & $24(82.8)$ & 0.355 \\
\hline From doctor & $16(51.6)$ & $18(62.1)$ & 0.414 \\
\hline From nurse & $26(83.9)$ & $24(82.8)$ & 0.908 \\
\hline From lactation counsellor & $14(45.2)$ & $15(51.7)$ & 0.611 \\
\hline
\end{tabular}


Table I. (Contd...)

\begin{tabular}{|c|c|c|c|}
\hline \multirow[t]{2}{*}{ Variable } & \multicolumn{2}{|c|}{ No. (\%) } & \multirow[t]{2}{*}{ p-value } \\
\hline & Regular expression $(n=31)$ & Routine breastfeeding $(n=29)$ & \\
\hline \multicolumn{4}{|l|}{ Birth factors } \\
\hline Normal delivery & $23(74.2)$ & $25(86.2)$ & 0.06 \\
\hline Instrumental delivery & $0(0)$ & $2(6.9)$ & \\
\hline Caesarean delivery & $8(25.8)$ & $2(6.9)$ & \\
\hline \multicolumn{4}{|l|}{ Perinatal support } \\
\hline Rooming-in & $10(32.3)$ & $11(37.9)$ & 0.645 \\
\hline Baby on breast after birth & $23(74.2)$ & $24(82.8)$ & 0.421 \\
\hline Breastfeeding within few hours of birth & $24(77.4)$ & $26(89.7)$ & 0.302 \\
\hline Breastfeeding problems encountered & $22(71.0)$ & $20(69.0)$ & 0.866 \\
\hline \multicolumn{4}{|l|}{ Infant factors } \\
\hline Required postnatal resuscitation & $1(3.2)$ & $2(6.9)$ & 0.606 \\
\hline Admission to NICU & $1(3.2)$ & $2(6.9)$ & \\
\hline Jaundice & $11(35.5)$ & $6(20.7)$ & \\
\hline
\end{tabular}

NICU: neonatal intensive care unit
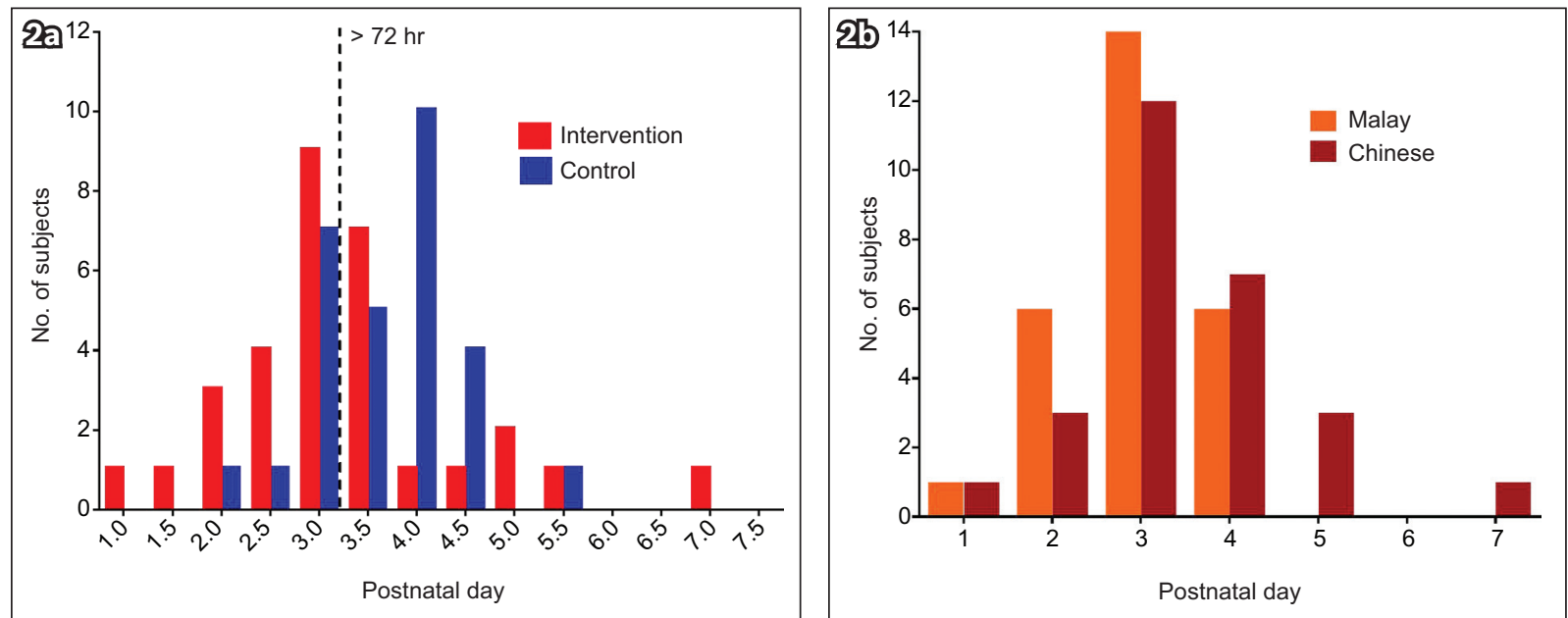

Fig. 2 Charts show a comparison of the first perception of breast fullness and copious milk flow between (a) the intervention group and control group; and (b) Malay and Chinese mothers.

groups fed a combination of breast milk and formula than breast milk alone/with non-milk fluids (intervention group: median 11 vs. $3, p=0.002$; control group: median 10 vs. $2, p=0.001$ ). Between the intervention and control groups, there was no significant difference in the number of women who had each feeding practice. The majority of women in the cohort breastfed at 2-3-hourly intervals, while one-third breastfed on demand (Fig. 4). Seven mothers breastfed at intervals of four hours or more. There were no significant differences between both groups in intervals of breastfeeding.

Overall, the mean difference in EBM volume for intervention versus control group was $73.9(95 \% \mathrm{Cl} 46.3,152.3) \mathrm{mL}$ on Day 3 and 225.2 (95\% Cl 98.1, 352.3) $\mathrm{mL}$ by Day 7 ( $<<0.02)$. There was significant interaction between EBM volumes and time (F-statistic $=2.28, p=0.049)$. In the first 48 hours, the mean EBM volume in the intervention group was similar to that of the control group, but it was significantly higher $(p<0.02)$ from Day 3 to Day 7 (99.3 [95\% Cl 46.3, 152.3] mL to 294.4
[95\% Cl 179.3, 409.5] $\mathrm{mL}$ ) compared to the control group (Day 3: 25.4 [95\% Cl 3.9, 46.9] mL to Day 7: 69.2 [95\% Cl 32.3, 106.1] mL) (Fig. 5a). Additionally, the mean total EBM volume over seven days was higher with intervention $(224.4[95 \% \mathrm{Cl}$ 97.4, 351.3] mL). Similarly, we noted a significant interaction between EBM volume fed to infants and time (F-statistic $=2.41$, $p=0.039$ ), which was similar between the groups in the first 48 hours and appeared higher in the intervention group from Day 3 to Day 7 (difference between intervention and control: 67.5 [95\% Cl 14.6, 120.4] $\mathrm{mL}$ to 156.9 [95\% Cl 59.2, 254.5] $\mathrm{mL}$ ). Over seven days, the mean difference in EBM fed to infants between the intervention and control groups was 156.8 (95\% Cl 59.2, 254.4) $\mathrm{mL}$.

Milk formula was fed to infants in both groups to supplement breast-milk feeds at the mothers' discretion. Comparing the intervention and control groups, there was no significant difference in the mean daily volumes of formula $(108.0 \mathrm{~mL}$ vs. $130.9 \mathrm{~mL}$, Fig. 5b) or the total daily volumes of breast milk 

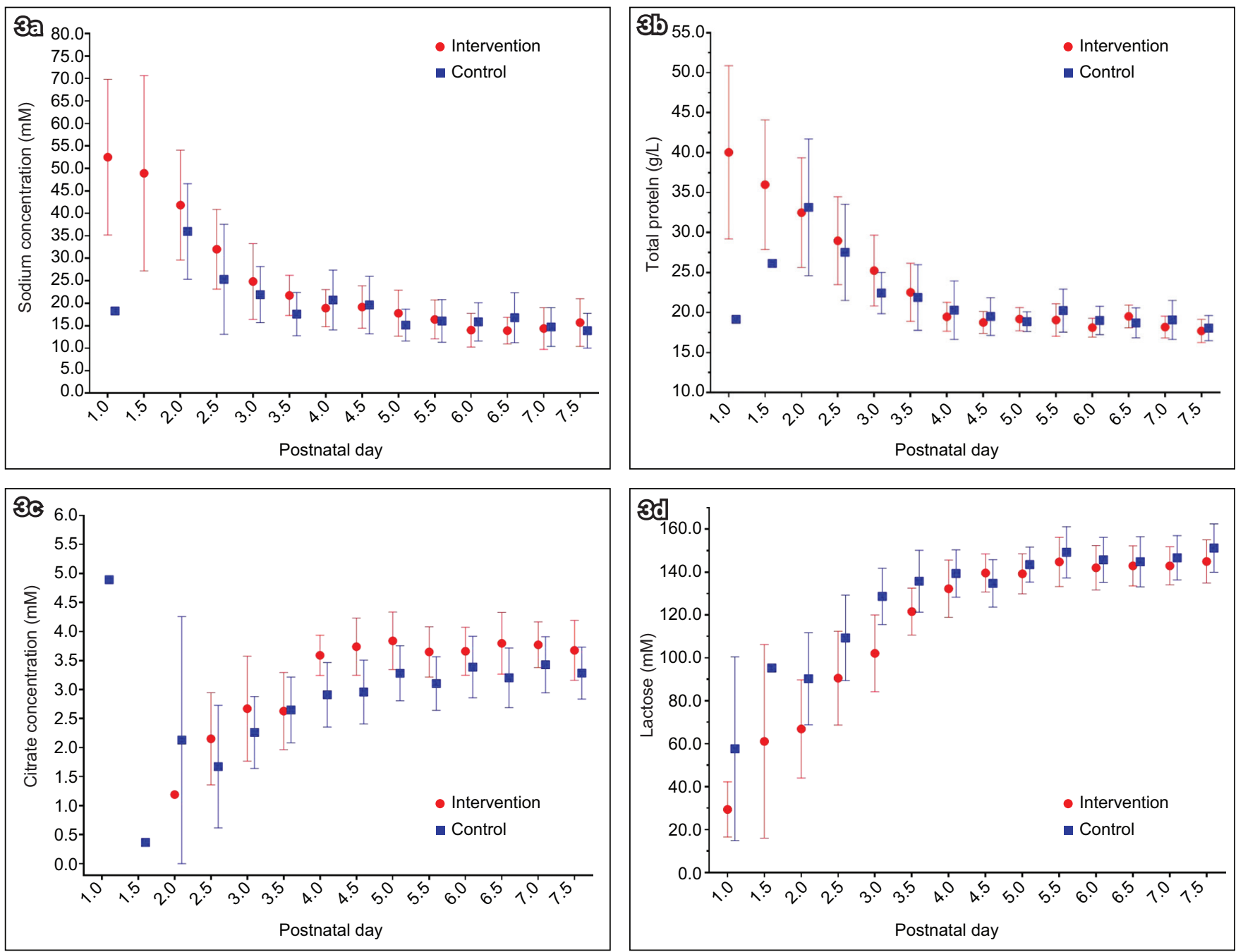

Fig. 3 Charts show a comparison of breast milk concentrations of (a) sodium; (b) total protein; (c) citrate; and (d) lactose between the intervention and control groups.
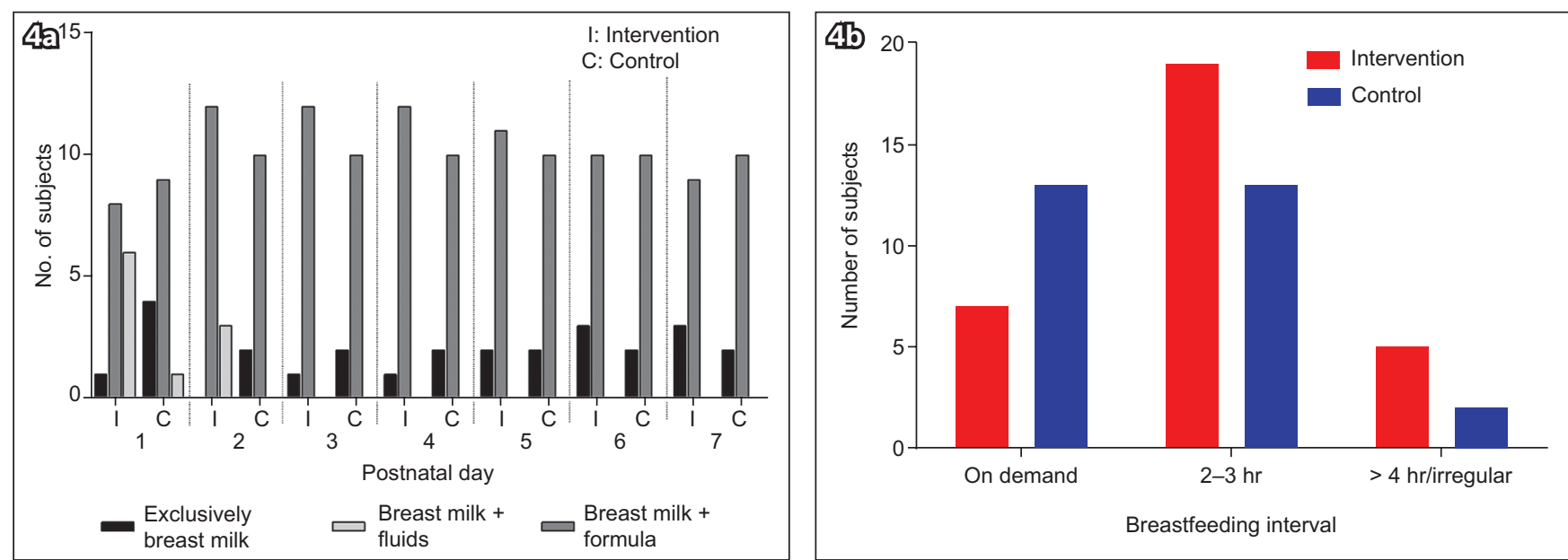

Fig. 4 Charts show a comparison of (a) breastfeeding practices and (b) intervals among mothers in the two groups.

and formula, which were $239.9(95 \% \mathrm{Cl} 153.1,326.7) \mathrm{mL}$ and 224.3 (95\% Cl 140.4, 308.2) mL, respectively. Daily total milk intake (EBM +/- formula) was similar at 239.9 (95\% Cl 153.1, $326.7) \mathrm{mL}$ and $224.3(95 \% \mathrm{Cl} 140.4,308.2) \mathrm{mL}$ in the intervention and control groups, respectively.
Babies were weighed at the start and end of the first postnatal week. There were no differences in mean weights between the intervention and control groups, which were 3,227 $(95 \% \mathrm{Cl}$ $3,094,3,359)$ g and 3,218 (95\% Cl 3,034, 3,402) g, respectively, on Day 2 (Fig. 5c). Weights increased over the first week in both 

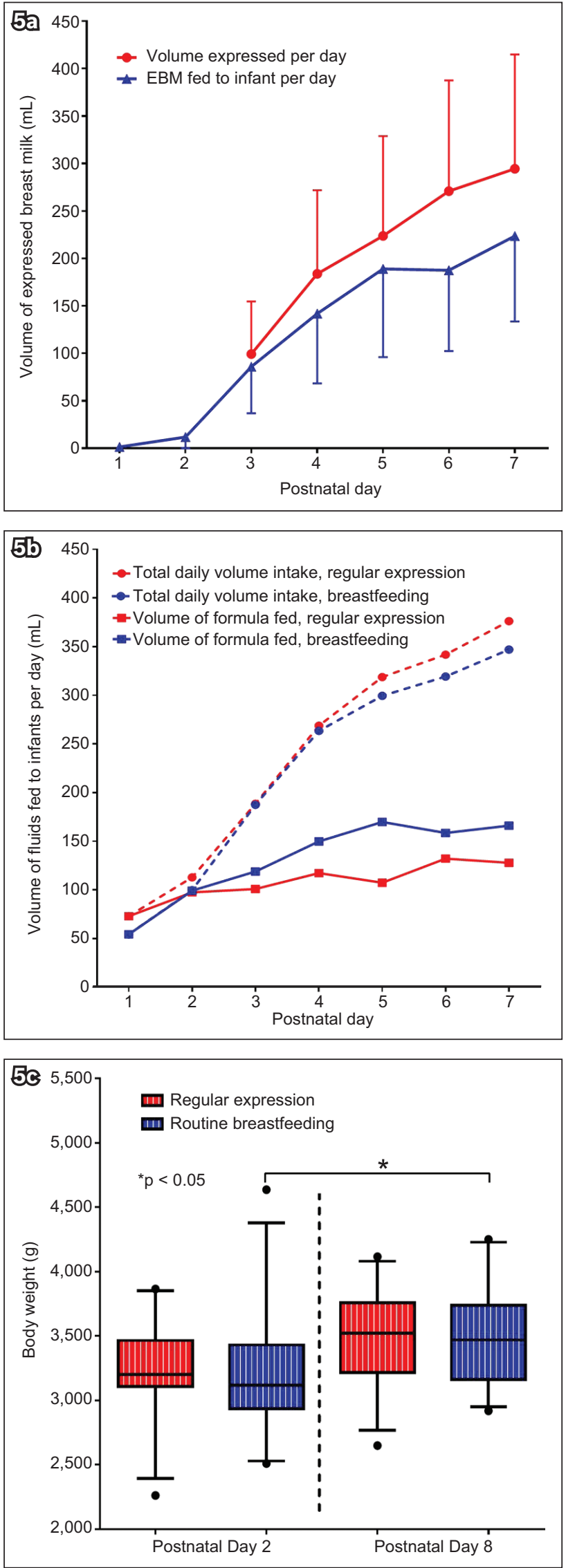

Fig. 5 Charts show (a) expressed breast milk (EBM) volume in the intervention group, who had regular milk expression; (b) comparison of formula feeds and total daily fluid volume fed in the intervention and control groups; and (c) comparison of mean infant weights in the two groups from Day 2 to Day 8. groups. On Day 8, mean weights in the intervention and control groups were $3,477(95 \% \mathrm{Cl} 3,334,3,621) \mathrm{g}$ and $3,479(95 \% \mathrm{Cl}$ $3,333,3,624) \mathrm{g}$ respectively. Only the increase in weight between Days 2 and 8 in the control group reached significance $(p=0.03)$.

\section{DISCUSSION}

In this study, the majority of women experienced voluminous flow on around the third to fourth postnatal days, an event often interpreted by nursing mothers as confirmation of successful breastfeeding initiation. Earlier onset Lall and a trend towards a lower incidence of DLII was observed in mothers performing a simple intervention of regular breast-milk expression using a standard EBP compared to unscheduled expression.

All mothers had copious flow by the end of Day 5, with the exception of one patient. Control group mothers reported copious flow by Day 4 . The biochemical profile of the breast milk demonstrated the expected changes that indicate Lall, with decreasing sodium and total protein, and increasing citrate and lactose. Biochemical steady states for each marker were reached around postnatal Day 4 in both groups, responding to clinical onset. While there is no significant difference in biochemical content between both groups on Day 4, earlier perception of lactogenesis, i.e. maternal perception of copious milk flow within the intervention group may result in greater confidence with establishing breast milk supply, a lower likelihood of supplementing feeds with formula, possibily resulting in a higher breastfeeding yield.

No ethnicity-specific breastfeeding practices were observed during the first week, but the number of participants reporting on breastfeeding practices was small. In support of other studies, our data confirms a direct correlation between milk volumes and frequency of expression and an inverse correlation with delivery-to-milk expression interval. ${ }^{(28)}$ Past studies have looked into the effects of breast pump suction patterns on the initiation and continuation of lactation for premature infants, ${ }^{(29)}$ as well as the usage of manual methods of milk expression coupled with electrical pumps for preterm infants. ${ }^{(30)}$ Examining breast milk expression and its frequency hence proves critical in furthering our understanding of breast milk supply for infants.

Mothers often encounter barriers to early direct breastfeeding, particularly when perinatal complications preclude early physical contact between mother and infant. ${ }^{(31,32)}$ Because early removal of breast milk is associated with successful transition to Lall, our data supports the clinical utility of scheduled expression for at-risk women. This proactive intervention is relatively easy, low cost and can be initiated with widely available equipment, while possibly avoiding pharmacological agents. It can be applied to women who are too ill to breastfeed after delivery, to enable lactation initiation in preparation for breastfeeding when they recover, and to mothers of multiples, preterm or low-birth-weight infants at higher risk of breastfeeding failure. ${ }^{(33)}$ Encouraging such a practice would be helpful in communities with suboptimal breastfeeding initiation and where early cessation is prevalent due to maternal perception of insufficient milk. Although this intervention might not appeal to every mother who intends to breastfeed, 
with judicious antenatal education and proper case selection, a protocol of early and regular breast milk expression can be a suitable addition to a healthcare policy promoting breastfeeding, giving women an acceptable alternative to initiating Lall if direct early breastfeeding is not feasible. There are few contraindications to using a breast pump if the mother or her caregiver is physically able to perform the necessary manoeuvres. Possible obstacles to widespread implementation of this practice include access to and the expense of acquiring hospital-grade EBPs with a double collection kit, ${ }^{(34)}$ since simultaneous breast emptying is more efficient than consecutive single-breast emptying. ${ }^{(35)}$

An important factor influencing the smooth transition from lactogenesis I (gestational development of mammary lactocytes) to Lall that this practice emulates is the early removal of breast milk, acting in concert with progesterone withdrawal and other perinatal factors. ${ }^{(36,37)}$ Early initiation of breastfeeding within the first half hour of life is the fourth step in the Baby-Friendly Hospital Initiative criteria, ${ }^{(38)}$ and is a critical strategy in the effort to elevate breastfeeding rates, contributing to significantly higher total milk production and earlier Lall in mothers of very-low-birth-weight babies. ${ }^{(19)}$ This is in contrast to the decreased milk production associated with late initiation (up to six days postpartum) in a similar cohort. ${ }^{(28)}$ Another important contributor is complete breast emptying. Maternal milk production and the breast's storage capacity both respond closely to the infant's demand for milk and completeness of breast emptying. Proper breast drainage also improves milk production efficacy, by increasing the capacity of the breast to produce milk in excess of the volume needed to satisfy the infant's hunger. ${ }^{(39)}$ Studies of milk production in dairy animals and humans have identified a local inhibitory factor that caused reversible disruption of milk flow, via local feedback inhibition, in a dose-dependent manner in the absence of effective milk removal, subsequently contributing to failed Lall. ${ }^{(37,40)}$

Encouraging sustained and exclusive breastfeeding following international recommendations is a key strategy in national programmes aimed at addressing inequalities in health and improving population well-being. ${ }^{(41,42)}$ The difficulty of sustaining this practice is underlined by the decline in breastfeeding rates over the course of the neonatal period. While initiation rates were encouragingly high, from 74\% in the United States (US, 2006 data) and $83 \%$ across the UK (2010 data), to 99\% in Singapore (2013 data), continued breastfeeding of any type declined steeply by six months to reach $42 \%$ in Singapore (EBF, $1 \%$ ), $43.5 \%$ in the US (EBF, $14.1 \%$ ) and $25 \%$ across the UK (EBF, negligible). ${ }^{(2,3,41,43,44)}$ There is an unfulfilled opportunity for obstetric healthcare providers to support breastfeeding women by offering a safe and effective intervention that may positively impact the quality and duration of breastfeeding.

Among the strengths of this study are that it provides useful information about the expected breast milk volume in our local Asian population consisting predominantly of Malay and Chinese women, which are substantially lower than reference volumes derived from relatively homogenous Caucasian populations. Mean EBM volume by Day 5 was around $220 \mathrm{~mL} /$ day, and total fluid volumes consumed by infants approximated $230 \mathrm{~mL} /$ day.
The regaining and surpassing of birth weight within the first week demonstrate that these infants were not compromised by lower milk consumption and should reassure Asian mothers that their breastfeeding efforts are likely to be sufficient.

The limitations of this study include not being able to accurately gauge the amount of breast milk consumed by breastfed infants in the control group, as we were not able to test weigh the infants after feeds. This data would have informed us about the true volume intake of breastfed infants. Furthermore, these interventions were designed not to interfere with the normal postnatal activities of trial participants; thus, we have several incomplete data sets because collection of samples and recording of data were not strictly enforced. Additionally, the data set is incomplete, as several mothers were uncontactable after they were recruited. Mothers were also not surveyed about their satisfaction with their individual breastfeeding experience, which would serve as a plausible research focus for future papers.

In conclusion, early and regular breast milk expression significantly contributes to the timely establishment of Lall, probably through mechanisms involving frequent and complete removal of intramammary inhibitors of breast milk production. This was supported by appropriate changes in biochemical markers by Day 3 in both groups. This simple intervention may be beneficial to women who are at risk of delayed Lall and premature breastfeeding cessation, and may contribute to promoting breastfeeding rates in industrialised countries.

\section{ACKNOWLEDGEMENT}

This study was funded by the National Healthcare Group grant NHG RPR 04027.

\section{REFERENCES}

1. World Health Organization. Proposed global targets for maternal, infant and young child nutrition. WHO discussion paper (6 February 2012). Available at: http://www.who.int/nutrition/events/2012_proposed_globaltargets_ backgroundpaper.pdf. Accessed January 102017.

2. UNICEF United Kingdom. UK Breastfeeding rates in the UK. Available at: https:// www.unicef.org.uk/babyfriendly/what-is-baby-friendly/breastfeeding-in-the-uk/ breastfeeding-rates-in-the-uk/. Accessed January 6, 2017

3. Chua L, Win AM. Prevalence of breastfeeding in Singapore. In: Statistics Singapore Newsletter September 2013 [online]. Available at: https://www.singstat.gov.sg/ docs/default-source/default-document-library/publications/publications_and_ papers/health/ssnsep13-pg10-14.pdf. Accessed December 20, 2016.

4. Victora CG, Bahl R, Barros AJ, et al; Lancet Breastfeeding Series Group. Breastfeeding in the 21st century: epidemiology, mechanisms, and lifelong effect. Lancet 2016; 387:475-90.

5. Shamir R. The benefits of breast feeding. Nestle Nutr Inst Workshop Ser 2016; 86:67-76.

6. Pang WW, Hartmann PE. Initiation of human lactation: secretory differentiation and secretory activation. J Mammary Gland Biol Neoplasia 2007; 12:211-21.

7. Arthur PG, Smith M, Hartmann PE. Milk lactose, citrate, and glucose as markers of lactogenesis in normal and diabetic women. J Pediatr Gastroenterol Nutr 1989; 9:488-96.

8. Neville MC, Keller R, Seacat J, et al. Studies in human lactation: milk volumes in lactating women during the onset of lactation and full lactation. Am J Clin Nutr 1988; 48:1375-86.

9. Chapman DJ, Pérez-Escamilla R. Identification of risk factors for delayed onset of lactation. J Am Diet Assoc 1999; 99:450-4; quiz 455-6.

10. Dewey KG, Nommsen-Rivers LA, Heinig MJ, Cohen RJ. Risk factors for suboptimal infant breastfeeding behavior, delayed onset of lactation, and excess neonatal weight loss. Pediatrics 2003; 112(3 Pt 1):607-19.

11. Rasmussen KM, Hilson JA, Kjolhede CL. Obesity may impair lactogenesis II. J Nutr 2001; 131:3009S-11S. 
12. Neubauer SH, Ferris AM, Chase CG, et al. Delayed lactogenesis in women with insulin-dependent diabetes mellitus. Am J Clin Nutr 1993; 58:54-60.

13. Cregan MD, De Mello TR, Kershaw D, McDougall K, Hartmann PE. Initiation of lactation in women after preterm delivery. Acta Obstet Gynecol Scand 2002 81:870-7.

14. Zanardo V, Savona V, Cavallin F, et al. Impaired lactation performance following elective delivery at term: role of maternal levels of cortisol and prolactin. J Matern Fetal Neonatal Med 2012; 25:1595-8.

15. Zanardo V, Pigozzo A, Wainer G, et al. Early lactation failure and formula adoption after elective caesarean delivery: cohort study. Arch Dis Child Fetal Neonatal Ed 2013; 98:F37-41.

16. Brownell E, Howard CR, Lawrence RA, Dozier AM. Delayed onset lactogenesis II predicts the cessation of any or exclusive breastfeeding. J Pediatr 2012; 161:608-14.

17. Chapman DJ, Pérez-Escamilla R. Does delayed perception of the onset of lactation shorten breastfeeding duration? J Hum Lact 1999; 15:107-11; quiz 137-9.

18. Sözmen M. Effects of early suckling of cesarean-born babies on lactation. Biol Neonate 1992; 62:67-8.

19. Parker LA, Sullivan S, Krueger C, Kelechi T, Mueller M. Effect of early breast milk expression on milk volume and timing of lactogenesis stage II among mothers of very low birth weight infants: a pilot study. J Perinatol 2012; 32:205-9.

20. Dozier AM, Howard CR, Brownell EA, et al. Labor epidural anesthesia, obstetric factors and breastfeeding cessation. Matern Child Health J 2013; 17:689-98.

21. Brown A, Jordan S. Impact of birth complications on breastfeeding duration: an internet survey. J Adv Nurs 2013; 69:828-39.

22. Rowe-Murray HJ, Fisher JR. Operative intervention in delivery is associated with compromised early mother-infant interaction. BJOG 2001; 108:1068-75.

23. Rowe-Murray HJ, Fisher JR. Baby friendly hospital practices: cesarean section is a persistent barrier to early initiation of breastfeeding. Birth 2002; 29:124-31.

24. Zanardo V, Trevisanuto D, Freato F. Maternal anxiety impairs lactation initiation and maintenance. Pediatrics 2006; 117:1859-60; author reply 1860-1.

25. Pang WW, Aris IM, Fok D, et al; GUSTO Study Group. Determinants of breastfeeding practices and success in a multi-ethnic Asian population. Birth 2016; 43:68-77.

26. Forster DA, Johns HM, McLachlan HL, et al. Feeding infants directly at the breast during the postpartum hospital stay is associated with increased breastfeeding a 6 months postpartum: a prospective cohort study. BMJ Open 2015; 5:e007512

27. Chapman DJ, Pérez-Escamilla R. Maternal perception of the onset of lactation is a valid, public health indicator of lactogenesis stage II. J Nutr 2000; 130:2972-80.

28. Hopkinson JM, Schanler RJ, Garza C. Milk production by mothers of premature infants. Pediatrics 1988; 81:815-20.

29. Meier PP, Engstrom JL, Janes JE, Jegier BJ, Loera F. Breast pump suction pattern that mimic the human infant during breastfeeding: greater milk output in less time spent pumping for breast pump-dependent mothers with premature infants. J Perinatol 2012; 32:103-10.
30. Morton J, Hall JY, Wong RJ, et al. Combining hand techniques with electric pumping increases milk production in mothers of preterm infants. J Perinatol 2009; 29:757-64.

31. Awi DD, Alikor EA. Barriers to timely initiation of breastfeeding among mothers of healthy full-term babies who deliver at the University of Port Harcourt Teaching Hospital. Niger J Clin Pract 2006; 9:57-64.

32. Cakmak H, Kuguoglu S. Comparison of the breastfeeding patterns of mothers who delivered their babies per vagina and via cesarean section: an observational study using the LATCH breastfeeding charting system. Int J Nurs Stud 2007; 44:1128-37.

33. Hwang SS, Barfield WD, Smith RA, et al. Discharge timing, outpatient followup, and home care of late-preterm and early-term infants. Pediatrics 2013; 132:101-8.

34. Meier PP. Breastfeeding in the special care nursery. Prematures and infants with medical problems. Pediatr Clin North Am 2001; 48:425-42.

35. Jones E, Dimmock PW, Spencer SA. A randomised controlled trial to compare methods of milk expression after preterm delivery. Arch Dis Child Fetal Neonatal Ed 2001; 85:F91-5.

36. Neville MC, Morton J. Physiology and endocrine changes underlying human lactogenesis II. J Nutr 2001; 131:3005S-8S.

37. Neville MC, Morton J, Umemura S. Lactogenesis. The transition from pregnancy to lactation. Pediatr Clin North Am 2001; 48:35-52.

38. UNICEF, World Health Organization. Baby-friendly hospital initiative: revised, updated and expanded for integrated care. Section 1: background and implementation. 2009. Available at: http://whqlibdoc.who.int/ publications/2009/9789241594967_eng.pdf. Accessed November 11, 2016.

39. Kent JC, Mitoulas L, Cox DB, Owens RA, Hartmann PE. Breast volume and milk production during extended lactation in women. Exp Physiol 1999; 84:435-47.

40. Peaker M, Wilde CJ. Feedback control of milk secretion from milk. J Mammary Gland Biol Neoplasia 1996; 1:307-15.

41. United States Breastfeeding Committee. Healthy People 2020: breastfeeding objectives. Available at: http://www.usbreastfeeding.org/p/cm/ld/fid=221. Accessed Januarary 4, 2017.

42. Department_of_Health, UK. National service framework for children, young people and maternity services: maternity services. Available at: https://www. gov.uk/government/uploads/system/uploads/attachment_data/file/199957/ National_Service_Framework_for_Children_Young_People_and_Maternity_ Services_-_Maternity_Services.pdf. Accessed October 20, 2016.

43. Foo LL, Quek SJ, Ng SA, Lim MT, Deurenberg-Yap M. Breastfeeding prevalence and practices among Singaporean Chinese, Malay and Indian mothers. Health Promot Int 2005; 20:229-37.

44. Bolling K, Grant C, Hamlyn B, Thornton A. Infant feeding survey 2005. Available at: http://digital.nhs.uk/catalogue/PUB00619. Accessed September 20, 2017. 